\title{
A low-power CMOS ASIC for X-ray Silicon Drift Detectors low-noise pulse processing
}

\author{
M. Ahangarianabhari, ${ }^{a}$ G. Bertuccio, ${ }^{a, 1}$ D. Macera, ${ }^{a}$ P. Malcovati, ${ }^{b}$ M. Grassi, ${ }^{b}$ A. \\ Rashevsky, ${ }^{c}$ I. Rashevskaya, ${ }^{c}$ A. Vacchi, ${ }^{c}$ G. Zampa, ${ }^{c}$ N. Zampa, ${ }^{c}$ F. Fuschino, ${ }^{d}$ \\ Y. Evangelista,${ }^{e}$ R. Campana,${ }^{d}$ C. Labanti ${ }^{d}$ and M. Feroci ${ }^{e}$ \\ ${ }^{a}$ Department of Electronics, Information and Bioengineering, Politecnico di Milano, \\ and INFN sez. Milano, Polo Territoriale di Como, \\ Via Anzani 42, 22100 Como, Italy \\ ${ }^{b}$ Department of Electrical Engineering, University of Pavia and INFN sez. Pavia, Via \\ A. Ferrata 5, 27100 Pavia, Italy \\ ${ }^{c}$ INFN sez. Trieste, \\ Padriciano 99, 34149 Trieste, Italy \\ ${ }^{d}$ INAF-IASF-Bologna, \\ Via Piero Gobetti 101, 40129 Bologna, Italy \\ 'INAF/IASF sez. Rome, \\ Via Fosso del Cavaliere 100, 00133 Roma, Italy \\ E-mail: Giuseppe.Bertuccio@polimi.it
}

RECEIVED: December 11, 2013

ACCEPTED: January 31, 2014

PUBLISHED: March 21, 2014

${ }^{1}$ Corresponding author. 


\section{Introduction}

The study and the development of Silicon Drift Detectors (SDDs) for high-resolution X-ray spectroscopy is a subject of research and growing interest [1-3]. The development of the SDDs technology, since its invention by Gatti and Rehak [4], made it possible to achieve high resolution X-ray spectroscopy and imaging because of their small read-out anode geometry and therefore their anode capacitance, which leads to reduction of the series noise contribution of the electronics in the detection system. Besides, the low anode current of the SDDs, attainable at temperatures below $-20^{\circ} \mathrm{C}$, makes it possible to achieve a very low noise detection system by keeping the parallel noise component at low levels. However, the achievable performance depends both on the quality of the detector as on the performance of the Front-End Electronics (FEE). Therefore, a proper design of the FEE circuit, based on Application Specific Integrated Circuit (ASIC), is required to achieve high-resolution spectroscopy.

In this paper we present the measurement results of a low-noise low-power Application Specific Integrated Circuit (ASIC), called VEGA-1, designed and optimized for analog pulse processing of signals from SDDs using the minimum possible electrical power, as required for application in space missions with detectors having several thousands of acquisition channels.

The specifications of the ASIC are described in section 2 . The SDD is described in the section 3. Section 4 presents a brief description about the VEGA-1 ASIC. The experimental results are presented in section 5 , and section 6 concludes the paper.

\section{Specifications of the VEGA-1 ASIC}

VEGA-1 was designed to fulfil all the requirements for reading out large-area multi-anode linear monolithic SDDs for X-Ray spectroscopy and imaging in an energy range from $500 \mathrm{eV}$ to $60 \mathrm{keV}$. The required energy resolution is better than $260 \mathrm{eV}$ FWHM at $6 \mathrm{keV}$, corresponding to an Equivalent Noise Charge (ENC) of FEE/detector system lower than 27 electrons r.m.s.. The reference 


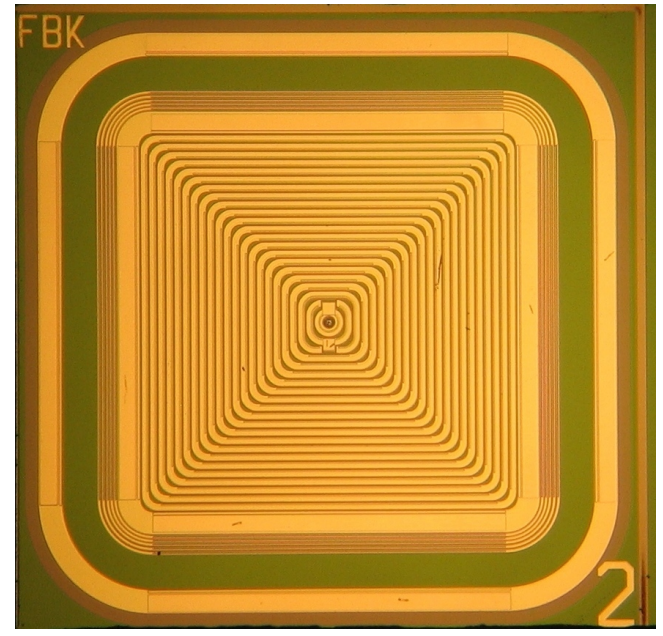

(a)

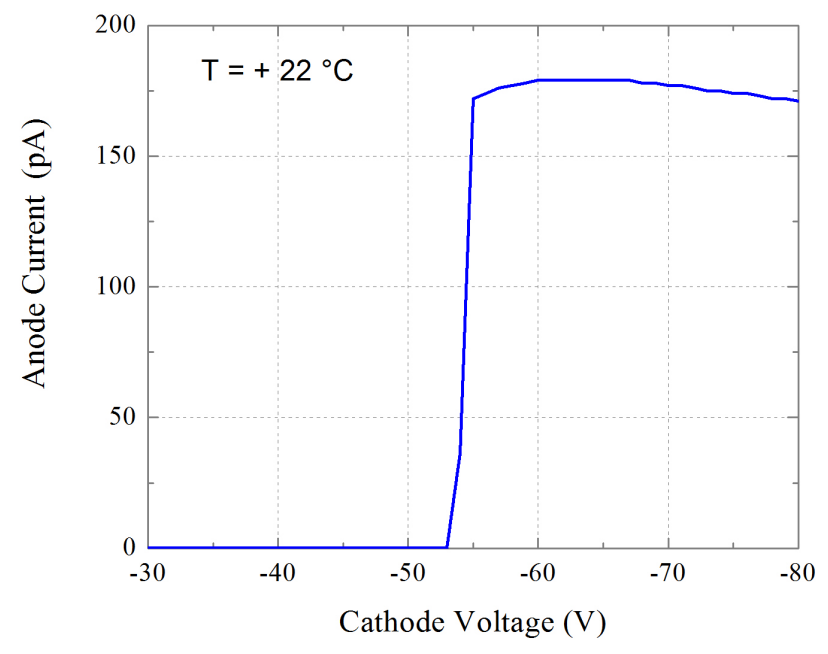

(b)

Figure 1. The $10 \mathrm{~mm}^{2}$ FBK quad-SDD. (a) Back (Anode) side of the SDD. (b) Readout anode leakage current at $22^{\circ} \mathrm{C}$ : the SDD is fully depleted at a bias voltage lower than $-55 \mathrm{~V}$.

SDDs for which VEGA-1 has been designed are $450 \mu \mathrm{m}$ thick, with an effective area of $76 \mathrm{~cm}^{2}$ read out by two rows of 112 anodes with a pitch of $970 \mu \mathrm{m}$ and an anode capacitance of about $350 \mathrm{fF}$. A detailed description of this detector, designed for the LOFT mission [5, 6], can be found in [7]. The aforementioned SDDs have a nominal anode current of about $0.7 \mathrm{pA}$ at $-32^{\circ} \mathrm{C}$, but the value can increase up to $10 \mathrm{pA}$ at the end of their life due to the radiation damage in the space mission. The detector is presently under construction but its potentialities have been demonstrated in previous prototypes [8]. The FEE must have less than $500 \mu \mathrm{W}$ total power consumption per channel in order to be suitable for its use in systems with a very large number of channels $\left(0.5 \times 10^{6}\right)$; at the same time, it shall accomplish all functionalities and requirements of an ADC-ready ASIC devoted to high-time-resolution X-ray spectroscopy.

\section{The Q10 Silicon Drift Detector}

The detector used for the measurements is a square SDD - named Q10-SDD - with a thickness of $450 \mu \mathrm{m}$ and an effective area of $10 \mathrm{~mm}^{2}$. The back (Anode) side of the detector is shown in figure 1 (a). A s mall $n^{+} \mathrm{p}$ ad in the detector c enter forms the read-out a node. An integrated voltage divider is implemented in the SDD in order to generate the proper biasing potentials for the cathodes and consequently forming the drift field. The innermost drift cathode, which is biased at a separate voltage, shields the anode from the noise of the drift cathode chain. The front $(\mathrm{P})$ side of the SDD is a uniform $p^{+}$implant constituting the entrance window for the radiation. The detector, designed at INFN, has been fabricated at FBK in Trento [9]. Figure 1(b) shows the measured readout anode leakage current of the aforementioned SDD as a function of the back side voltage, while the front side was left floating. By increasing the bias voltage of the outmost cathode, the depleted volume advances from the back side to the front side, and reaches the front 


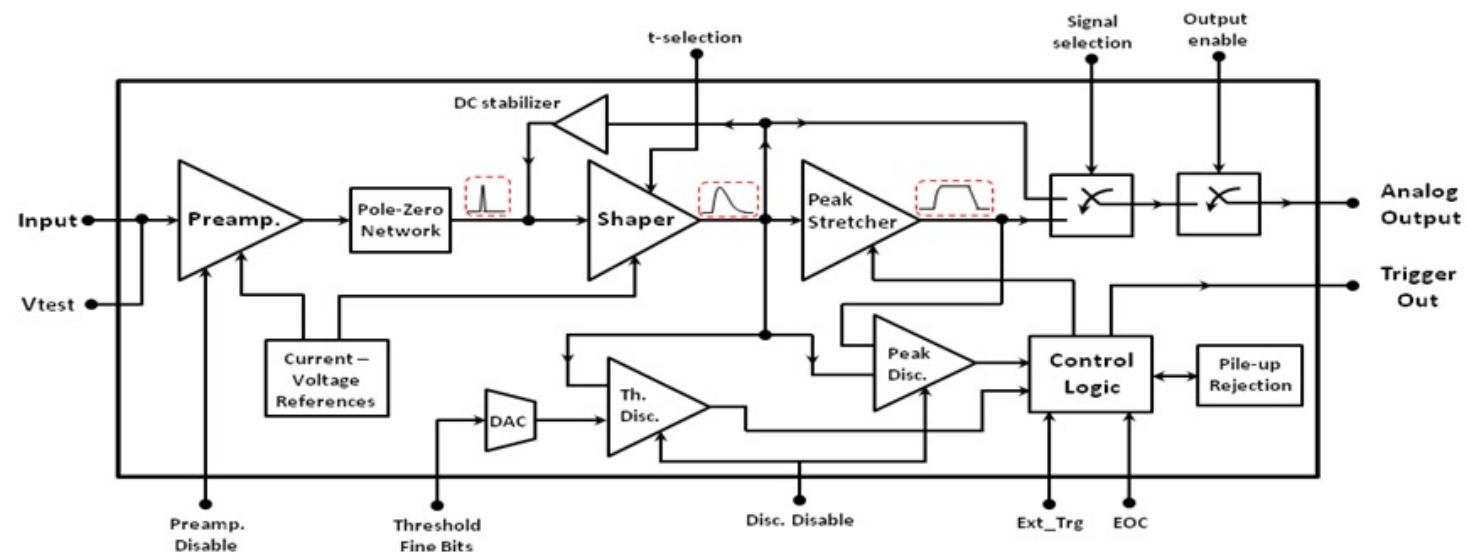

Figure 2. Block diagram of the read out FEE circuit.

side at $V_{\text {bias }}=-55 \mathrm{~V}$. The measured anode current is about $180 \mathrm{pA}$ at $+22^{\circ} \mathrm{C}$ when the SDD is fully depleted, which corresponds to a current density of $1.8 \mathrm{nA} / \mathrm{cm}^{2}$.

\section{The VEGA-1 ASIC}

The VEGA-1 ASIC has been designed in $0.35-\mu \mathrm{m}$ CMOS Technology and manufactured at Austriamicrosystems [10]. This technology offers four metal layers, two polysilicon layers and highresistive poly $(1.2 \mathrm{k} \Omega / \square)$, thus allowing the required integration density. The low-noise characteristics of the MOSFETs in this technology have been studied and proved in previous works [11, 12].

Figure 2 shows the block diagram of the VEGA-1 ASIC, consisting of an analog and a digital/mixed-signal section. The analog section includes a low-noise charge sensitive preamplifier, a R C-CR s haper w ith digitally s electable s haping times from $1.6 \mu$ s to $6.6 \mu \mathrm{s}$, and a peak stretcher/sample-and-hold circuit. The digital/mixed-signal section includes an amplitude discriminator with coarse and fine threshold 1 evel s ettings, and a peak d iscriminator. A 1 ogic circuit performs pile-up rejection, signal sampling, trigger generation, channel reset, and controls the ASIC configuration, including the possibility to disable the preamplifier and the discriminator. The VEGA-1 has been designed and manufactured in single and 32-channel versions with dimensions of $200 \mu \mathrm{m} \times 500 \mu \mathrm{m}$ per channel. Full details on the design and the functionalities of the VEGA-1 ASIC are presented in [13].

\section{Experimental results}

A photograph of the VEGA-1 bonded to the Q10-SDD is shown in figure 3. The ASIC is placed on top of the SDD in order to minimize the distance between the input pad of the ASIC and the anode of the SDD, thus minimizing the stray capacitances at the ASIC input: in this way the series noise contribution can be kept as low as possible. A kapton foil separates the ASIC back contact from the SDD for electrical isolation.

The VEGA-1 ASIC, together with the Q10-SDD, has been placed in a thermostatic chamber and tested with ${ }^{55} \mathrm{Fe}$ and ${ }^{241} \mathrm{Am}$ radiation sources at $-30^{\circ} \mathrm{C}$. Figure 4 shows the shaper output 


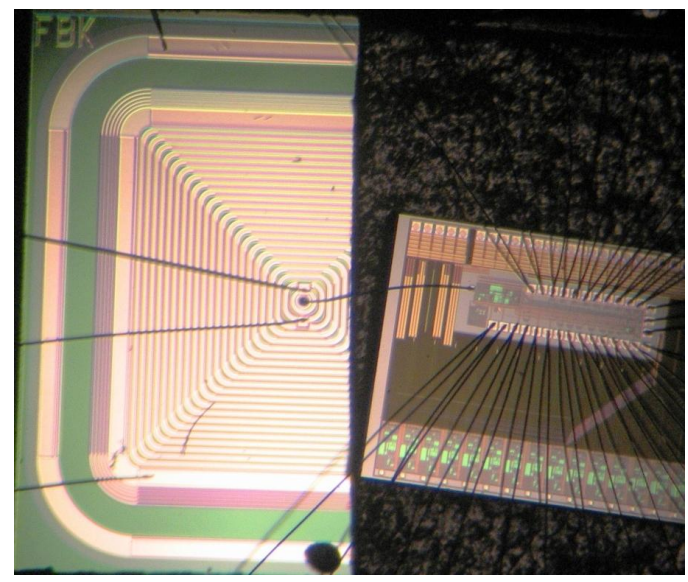

Figure 3. Photograph of the VEGA-1 ASIC bonded to the $10 \mathrm{~mm}^{2}$ FBK SDD. A kapton foil separates the ASIC from the SDD.

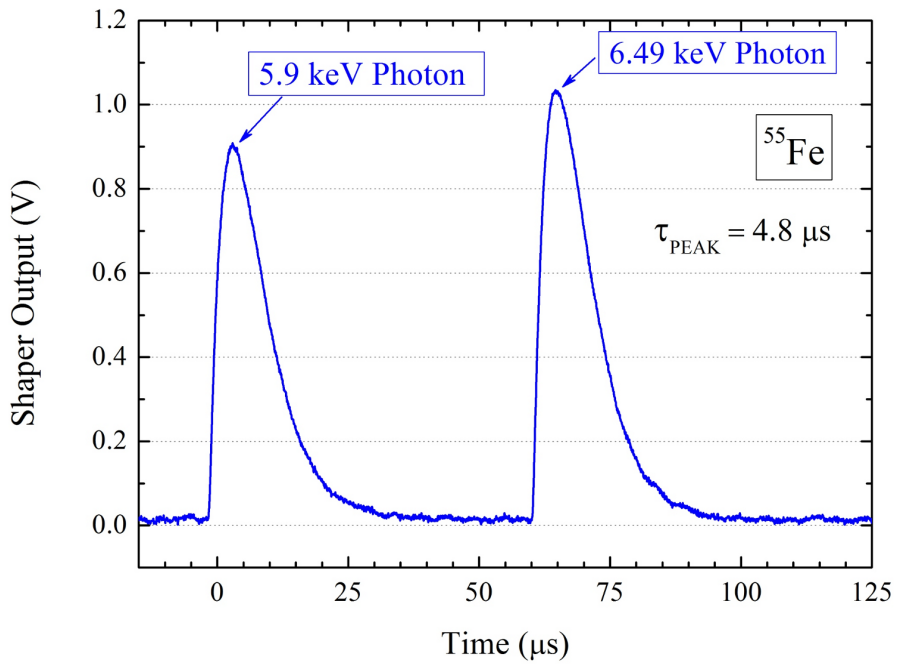

Figure 4. Measured shaper output with SDD of $10 \mathrm{~mm}^{2}$ at $-30^{\circ} \mathrm{C}$ and $4.8 \mu$ s peaking time. The two peaks correspond to $5.9 \mathrm{keV}$ and $6.49 \mathrm{keV}$ photon energies of ${ }^{55} \mathrm{Fe}$ radiation source.

signal resulting from the acquisition of two photons at $5.9 \mathrm{keV}$ and $6.49 \mathrm{keV}$ from ${ }^{55} \mathrm{Fe}$. Since the ASIC has a dynamic range of $60 \mathrm{keV}$, a very low-noise amplifier has been used at the output of the ASIC in order to amplify the signal by a factor of 10 and monitor it on the oscilloscope operating in single shot triggering mode. This acquisition highlights the high signal to noise ratio of the system.

Figure 5 shows the ${ }^{55} \mathrm{Fe}$ spectrum acquired at $-30^{\circ} \mathrm{C}$ and $3.6 \mu$ s peaking time. The pulser is set at $450 \mathrm{eV}$ and the measured energy resolution is $140 \mathrm{eV} \mathrm{FWHM,} \mathrm{corresponding} \mathrm{to} \mathrm{an} \mathrm{ENC} \mathrm{of}$ 16 electrons r.m.s. . The line width of the ${ }^{55} \mathrm{Fe} \mathrm{Mn} \mathrm{K}_{\alpha}$ line is $185 \mathrm{eV}$ FWHM, well below the upper limit of $260 \mathrm{eV}$ required by the project specifications, although the LOFT SDD has a larger anode capacitance and so a higher noise is expected.

Figure 6(a) shows the ${ }^{241} \mathrm{Am}$ spectrum acquired at $-30^{\circ} \mathrm{C}$ and $3.6 \mu$ s peaking time. In this measurement, the pulser is set at $500 \mathrm{eV}$ and has a measured line width of $163 \mathrm{eV}$ FWHM. Figure 6(b) shows the ${ }^{241} \mathrm{Am}$ spectrum of figure 6(a) in more details. As it can be seen, all important 


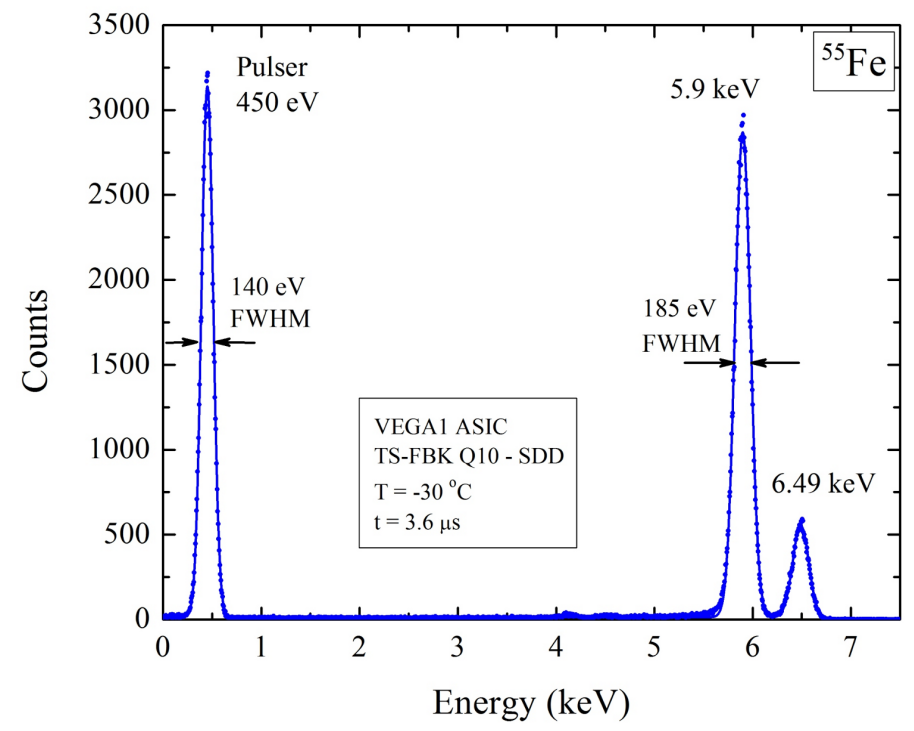

Figure 5. ${ }^{55} \mathrm{Fe}$ spectrum acquired with SDD of $10 \mathrm{~mm}^{2}$ at $-30^{\circ} \mathrm{C}$ and $3.6 \mu$ s peaking time. The measured energy resolution of the pulser is $140 \mathrm{eV}$ FWHM, corresponding to the ENC of 16 electrons r.m.s. .

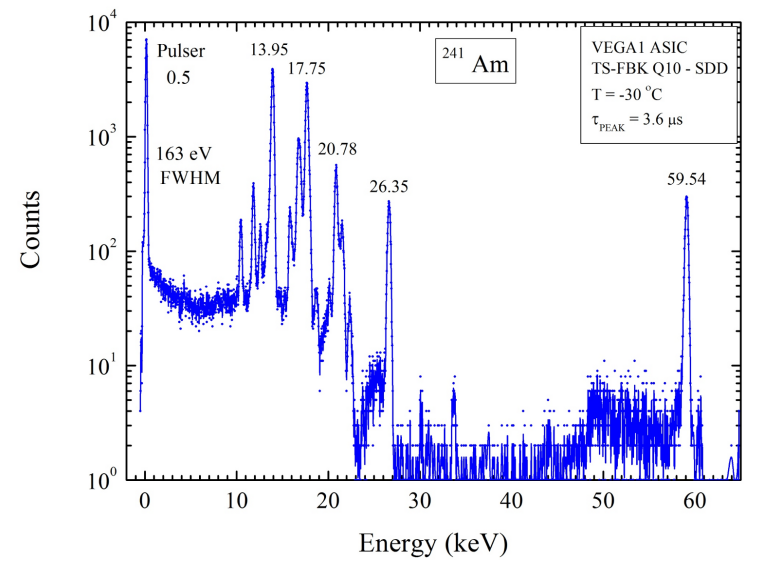

(a)

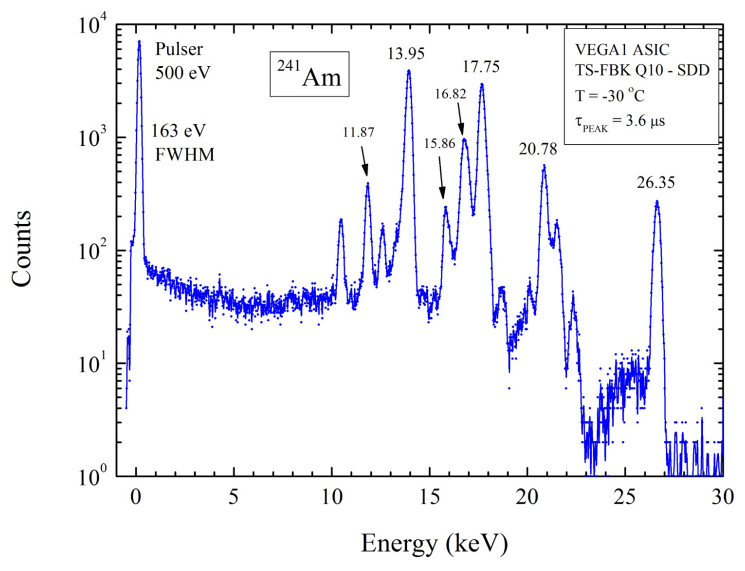

(b)

Figure 6. ${ }^{241} \mathrm{Am}$ spectrum acquired with SDD of $10 \mathrm{~mm}^{2}$ at $-30^{\circ} \mathrm{C}$ and $3.6 \mu$ s peaking time. An energy resolution of $163 \mathrm{eV}$ FWHM is measured on the pulser line. (a) The acquired ${ }^{241} \mathrm{Am}$ spectrum up to $60 \mathrm{keV}$. (b) Focus of the spectrum of the left figure up to $30 \mathrm{keV}$.

energy lines of ${ }^{241} \mathrm{Am}$ are completely visible. An energy resolution of about $262 \mathrm{eV}$ FWHM is measured at the $13.95 \mathrm{keV}$ line of ${ }^{241} \mathrm{Am}$. This measurement also proves the required operative energy range ( $500 \mathrm{eV}$ to $60 \mathrm{keV})$ of the VEGA-1 ASIC.

Figure 7 shows the measured linearity and linearity error determined from the mono-energetic lines of the ${ }^{241} \mathrm{Am}$ spectrum of figure $6(\mathrm{a})$, deriving a linearity error between $-0.7 \%$ and $+1 \%$ in the $13-59 \mathrm{keV}$ energy range.

Figure 8 shows the comparison between the measured ENC of the VEGA-1 ASIC without and with the detector connected. A minimum ENC of 16 electrons r.m.s. (equivalent to $140 \mathrm{eV} \mathrm{FWHM)}$ 


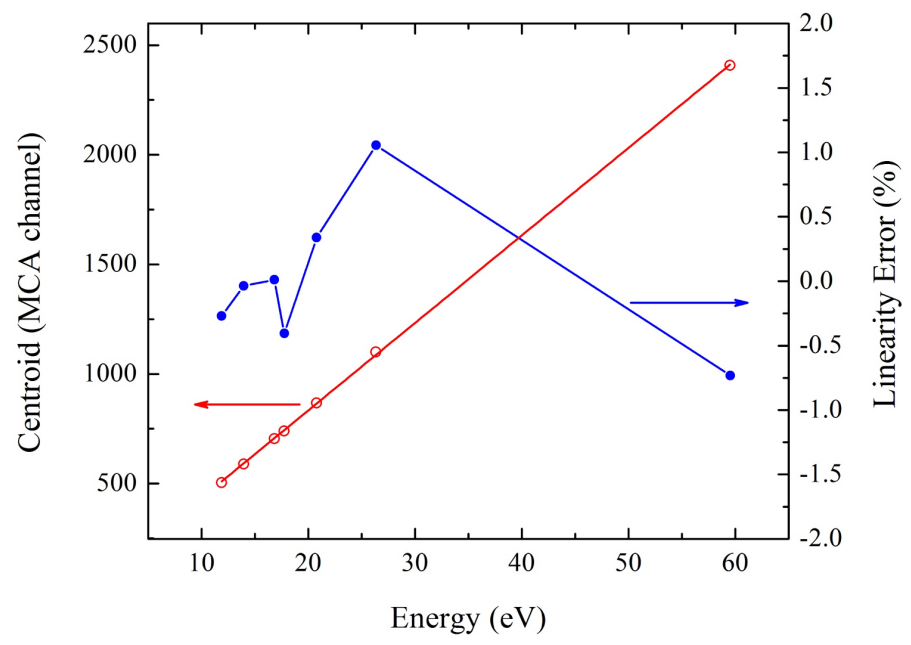

Figure 7. Measured linearity (red) and linearity error (blue) of the whole FEE circuit loaded with $10 \mathrm{~mm}^{2}$ FBK quad SDD for ${ }^{241} \mathrm{Am}$ spectrum of figure 7 at $3.6 \mu$ s peaking time and $-30^{\circ} \mathrm{C}$.

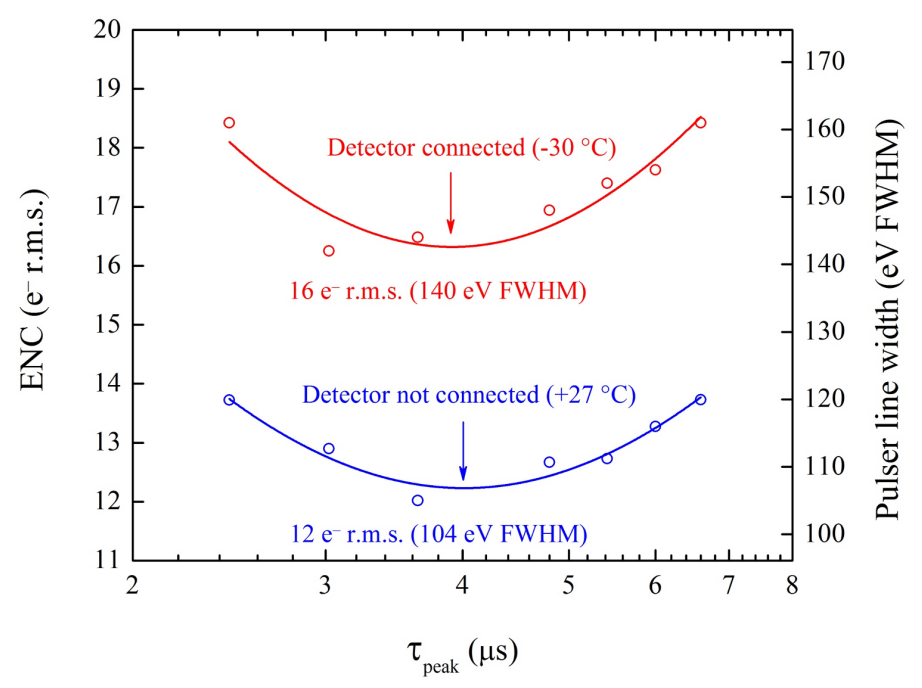

Figure 8. The measured noise of the FEE circuit before (blue line) and after (red line) bonding to the detector. A minimum intrinsic ENC of about 12 electrons r.m.s. at $3.6 \mu$ s shaping time and $+27^{\circ} \mathrm{C}$ is measured for the FEE. A minimum ENC of about 16 electrons r.m.s. at $3.0 \mu$ s shaping time and $-30^{\circ} \mathrm{C}$ is measured for the FEE bonded to the $10 \mathrm{~mm}^{2}$ FBK quad SDD.

is measured at $-30^{\circ} \mathrm{C}$ and $3.0 \mu$ s peaking time when the FEE input is connected to the SDD; whereas, the measured minimum intrinsic noise of the ASIC without detector is 12 electrons r.m.s. (equivalent to $104 \mathrm{eV} \mathrm{FWHM),} \mathrm{at} 3.6 \mu$ s peaking time and room temperature. The noise increment is due to the additional capacitance at the input of the ASIC (due to the anode capacitance of the SDD and the stray capacitance), as well as the leakage current of the detector.

A total power consumption of $482 \mu \mathrm{W}$ is measured for the single channel version of VEGA-1 ASIC. Since the implemented current/voltage references circuit is common to all the 32 channels in the multi-channel version, a total power consumption of about $420 \mu \mathrm{W}$ per channel is expected for the 32-channel version. 
Table 1. VEGA-1 ASIC and Quad-SDD features.

\begin{tabular}{|ll|}
\hline Parameter & Value \\
\hline Technology & AMS $0.35 \mu \mathrm{s}$ CMOS C35B4C3 \\
Input charge & Negative \\
Dynamic range & $500 \mathrm{eV}-60 \mathrm{keV}$ \\
Pulse shaping & $\mathrm{CR}-\mathrm{RC}$ \\
Shaping times & $1.6 \mu$ s to $6.6 \mu \mathrm{s}$ (3 bits selectable) \\
Linearity error & from $-0.7 \%$ to $1 \%$ \\
ENC (intrinsic, $\left.T=+27^{\circ} \mathrm{C}\right)$ & 12 electrons r.m.s. (without detector) \\
ENC (intrinsic, $\left.T=-30^{\circ} \mathrm{C}\right)$ & 16 electrons r.m.s. (with detector) \\
Voltage supplies $($ Analog) & $+3.3 \mathrm{~V},+2 \mathrm{~V}$ \\
Voltage supplies (Digital) & $+3.3 \mathrm{~V}$ \\
& $482 \mu \mathrm{W}$ (single channel version) \\
Power consumption & $420 \mu \mathrm{W} / \mathrm{ch}(32$ channel version) \\
SDD active area & $10 \mathrm{~mm}{ }^{2}$ \\
SDD thickness & $450 \mu \mathrm{m}$ \\
$I_{\text {ANODE }}$ & $\sim 180 \mathrm{pA} @+22^{\circ} \mathrm{C}$ \\
\hline
\end{tabular}

Table 1 summarizes the main experimental results of the VEGA-1 ASIC and also the characteristics of the Q10-SDD.

\section{Conclusion}

We have presented the experimental test of the VEGA-1 ASIC designed and manufactured for pulse processing of signals coming from monolithic silicon drift detectors. VEGA-1 performs high-resolution X-ray spectroscopy and imaging in the energy range from $500 \mathrm{eV}$ to $60 \mathrm{keV}$ with very low power consumption. A minimum ENC of 16 electrons r.m.s. at $3.0 \mu$ s peaking time and $-30^{\circ} \mathrm{C}$ is measured when connected to a $10 \mathrm{~mm}^{2} \mathrm{Q} 10-\mathrm{SDD}$ while the minimum intrinsic (without detector) ENC is 12 electrons r.m.s. at $3.6 \mu$ s peaking time and room temperature. A total power consumption of $482 \mu \mathrm{W}$ is measured in the single channel version of the ASIC, and about $420 \mu \mathrm{W}$ per channel is expected in the 32-channel version.

\section{Acknowledgments}

The financial s upport of the $\mathrm{N}$ ational Institute of N uclear Physics (XDXL e xperiments), Italian Space Agency (ASI) and National Institute of Astrophysics (INAF) is acknowledged. Authors thank S. Masci for PC board and the wire bonding of the ASIC and the SDD. 


\section{References}

[1] W. Chen et al., Development of low-resistivity silicon drift detector arrays for soft X-rays, IEEE NSS/MIC (2012) 931.

[2] G. Zampa et al., A room-temperature spectroscopic performance of a very-large area silicon drift detector, Nucl. Instrum. Meth. A 633 (2011) 15.

[3] P. Rehak et al., Arrays of silicon drift detectors for an extraterrestrial X-ray spectrometer, Nucl. Instrum. Meth. A 624 (2010) 260.

[4] E. Gatti and P. Rehak, Semiconductor drift chamber - an application of a novel charge transport scheme, Nucl. Instrum. Meth. 225 (1984) 608.

[5] M. Feroci et al., The Large Observatory for X-ray Timing (LOFT), Exp. Astron. 34 (2012) 415 [arXiv: 1107.0436].

[6] LOFT, a candidate ESA Cosmic Vision E3 mission, available at: http://www.isdc.unige.ch/loft/index.php/the-loft-mission.

[7] S. Zane et al., A large area detector proposed for the Large Observatory for X-ray Timing (LOFT), Proc. SPIE 8443 (2012) 84432F [arXiv : 1209.1498].

[8] G. Zampa, A. Rashevsky and A. Vacchi, The X-ray spectroscopic performance of a very large area silicon drift detector, IEEE Trans. Nucl. Sci. 56 (2009) 832.

[9] Fondazione Bruno Kessler (FBK), Trento, Italy.

[10] Austriamicrosystems AG, Tobelbaderstrasse 30, Schloss Premstaetten, A 8141 Unterpremstaetten, Austria, http://www.austriamicrosystems.com.

[11] G. Bertuccio and S. Caccia, Progress in ultra-low-noise ASICs for radiation detectors, Nucl. Instrum. Meth. A 579 (2007) 243.

[12] G. Bertuccio and S. Caccia, Noise minimization of MOSFET input charge amplifiers based on $\Delta \mu$ and $\Delta N 1 /$ f models, IEEE Trans. Nucl. Sci. 56 (2009) 1511.

[13] M. Ahangarianabhari, D. Macera, G. Bertuccio, P. Malcovati and M. Grassi, VEGA-1: a low-power front-end ASIC for large area multi-linear X-ray silicon drift detectors: design and first experimental characterization, to appear. 